Supporting Information for

\title{
Chemical-Pressure-Modulated $\mathrm{BaTiO}_{3}$ Thin Films with Large Spontaneous Polarization and High Curie Temperature
}

Yilin Wang, ${ }^{1,2}$ Linxing Zhang, ${ }^{1}$ Jiaou Wang, ${ }^{3}$ Qiang Li, ${ }^{1}$ Huanhua Wang, ${ }^{3}$ Lin Gu, ${ }^{4}$ Jun Chen, ${ }^{1}$ Jinxia Deng, ${ }^{1}$ Kun Lin, ${ }^{1}$ Ling Huang, ${ }^{2}$ and Xianran Xing ${ }^{1, *}$

${ }^{1}$ Beijing Advanced Innovation Center for Materials Genome Engineering, Institute of Solid State Chemistry, University of Science and Technology Beijing, Beijing 100083, China ${ }^{2}$ Institute of Advanced Materials (IAM), Jiangsu National Synergetic Innovation Center for Advanced Materials (SICAM), Nanjing Tech University, 30 South Puzhu Road, Nanjing 211816, China

${ }^{3}$ Institute of High Energy Physics, Chinese Academy of Sciences, Beijing 100049, China ${ }^{4}$ Beijing National Laboratory for Condensed Matter Physics, Institute of Physics, Chinese Academy of Sciences, Beijing 100190, China

*Corresponding author. E-mail: xing@,ustb.edu.cn.

\section{This PDF file includes:}

1. Methods.

2. Supplementary Figures S1-9.

3. Supplementary Table S1.

4. References List (1-10). 


\section{Methods}

Materials: The $\mathrm{BaTiO}_{3}$ composite thin films (c-BTO) are grown on $\mathrm{SrTiO}_{3}$ with $0.7 \mathrm{wt} \%$ $\mathrm{Nb}$ doping (100) substrates by RF sputtering using a $\mathrm{BaTiO}_{3}$ target with extra $\mathrm{Ba}$ content $(\mathrm{Ba} / \mathrm{Ti}$ $=1.5$ ), while the single-phase $\mathrm{BaTiO}_{3}$ thin films ( $s$-BTO) are grown with a pure $\mathrm{BaTiO}_{3}$ target. The basic vacuum before sputtering is $4 \times 10^{-3}$ mTorr. The substrate temperature of $c$-BTO is $650{ }^{\circ} \mathrm{C}$ in a pure Ar atmosphere with total pressure of 25 mTorr. The sputtering temperature of $s$-BTO is $600{ }^{\circ} \mathrm{C}$ in a mixture of $\mathrm{Ar} / \mathrm{O}$ with the ratio of $5 / 1$ and the total pressure is $10 \mathrm{mTorr}$. Both samples are post-annealed in the same temperature and atmosphere as the sputtering condition. These deposition conditions are optimized with the best remanent polarization of both $c$-BTO and $s$-BTO films.

Lattice Structure Characterization: The synchrotron-based XRD and RSM measurements were carried out at Diffuse X-ray Scattering Station of Beijing Synchrotron Facility (1W1A beamline). The $\theta-2 \theta$ scans of $c$-BTO and $s$-BTO were performed to obtain the out-of-plane lattice parameters of the films and substrates. X-ray reflectivity (XRR) was conducted to calculate the thickness of $c$-BTO and $s$-BTO. The phi scan of the (103) plane of $c$-BTO was scanned to reveal the symmetry. The high-resolution synchrotron-based reciprocal space mapping (RSM) were measured to show the detailed structure around the (103) plane. For the high-temperature RSMs and XRD, the samples are pasted with silver paste. And during the measurements, the $\mathrm{N}_{2}$ flow was applied for the protection to rule out the reaction of $\mathrm{BaTiO}_{3}$ or $\mathrm{BaO}$ in air.

Microstructure Characterization: The HAADF-STEM images were taken by an atomicresolution analytical microscope (JEM-ARM 200F). The cross-sectional sample was obtained by FIB. Previous to the FIB process, a Pt layer was sputtered onto the surface to protect the film. In Z-contrast HAADF-STEM, the brightness of atoms is proportional to $\sim Z^{1.7}$ ( $Z$ represents the atom number). In $c$-BTO, $\mathrm{Ba}(\mathrm{Z}=56)$ and $\mathrm{Ti}(\mathrm{Z}=22)$ columns could be easily distinguished due to the difference of brightness, which is beneficial for the distinguishing of 
$\mathrm{BaTiO}_{3}$ and $\mathrm{BaO}$. As shown in Figure 3d, the intensity profile displays the "B"-site columns, which is $\mathrm{Ba}$ in $\mathrm{BaO}$ with higher intensity and $\mathrm{Ti}$ in $\mathrm{BaTiO}_{3}$ with lower intensity, helping us to distinguish $\mathrm{BaTiO}_{3}$ and $\mathrm{BaO}$ in the STEM image.

Composition Characterization: Auger electron spectroscopy (AES) (PHI-710, ULVACPHI, Japan) with depth analysis was used to determine the component distribution in the outof-plane direction. The Ar ion sputtering was applied to remove the surface layer. Through increasing the stripping time, the deeper layer would be measured by Auger electron spectroscopy, and eventually getting the depth-dependent Auger electron spectroscopy. The spectra were measured after each sputtering sequence until reaching the substrate. The sputtering rate was nearly $2 \mathrm{~nm} / \mathrm{min}$. ICP and XPS were used to assist the determination of the composition and qualitatively compare the oxygen vacancies of $c$-BTO and $s$-BTO. The ICP samples are deposited on the (100)-oriented $\left(\mathrm{LaAlO}_{3}\right)_{0.29^{-}}\left(\mathrm{SrAl}_{0.5} \mathrm{Ta}_{0.5} \mathrm{O}_{3}\right)_{0.71}(\mathrm{LSAT})$ substrate, which possess the similar lattice parameters with $\mathrm{Nb}: \mathrm{STO}\left(a_{\mathrm{LSAT}}=3.868 \AA\right.$ and $a_{\mathrm{Nb}: \mathrm{STO}}=3.905$ $\AA$ ) and could rule out the Ti atoms in the Nb:STO. The $c$-BTO and $s$-BTO films on the LSAT substrates were then dissolved in HF solution with $1 \%$ concentration. In Table.S1, the ICP results shows the average content of $c$-BTO and $s$-BTO, indicating the extra Ba content in $c$ BTO.

Ferroelectric properties: The AFM and PFM experiments were carried out by MFP-3D, Asylum Research, with contacting mode. The AFM images were obtained using the AFM tip. The patterned PFM image was written by a d.c. voltage of $\pm 10 \mathrm{~V}$ via a conductive PFM tip. Then the reversed domain patterns were read. The dual frequency resonant-tracking technique (DART) was applied to improve the PFM sensitivity. The local piezoresponse loop measurements were conducted by applying a $10 \mathrm{~V}$ a.c. driven voltage. The ferroelectric loops were measured by a ferroelectric tester (TF-Analyzer 1000, aixACCT, Germany) with the assistance of the high-voltage accessory and the frequency of the voltage pulse is $1000 \mathrm{~Hz}$. The circular patterns of the top electrodes were projected via photolithography with a diameter of 
$20 \mu \mathrm{m}$. Then the direct current sputtering was applied to deposit the Pt electrode with a thickness of $100 \mathrm{~nm}$. The films were dissolved in acetone to remove the photoresist and finally the circular top electrodes were constructed. The PUND measurement is performed by TF-Analyzer 2000 with square wave function.

Electronic Structure Characterization: The XAS and XPS were collected at the photoelectron spectroscopy station of Beijing Synchrotron Radiation Facility (4B9B beamline). The photon energy was corrected via the Au standard sample to normalize the spectra of Ti $L$ edge and $\mathrm{O} K$-edge. 


\section{Supplementary Figures}

Thickness demonstration of TEM and X-ray reflectivity (XRR)
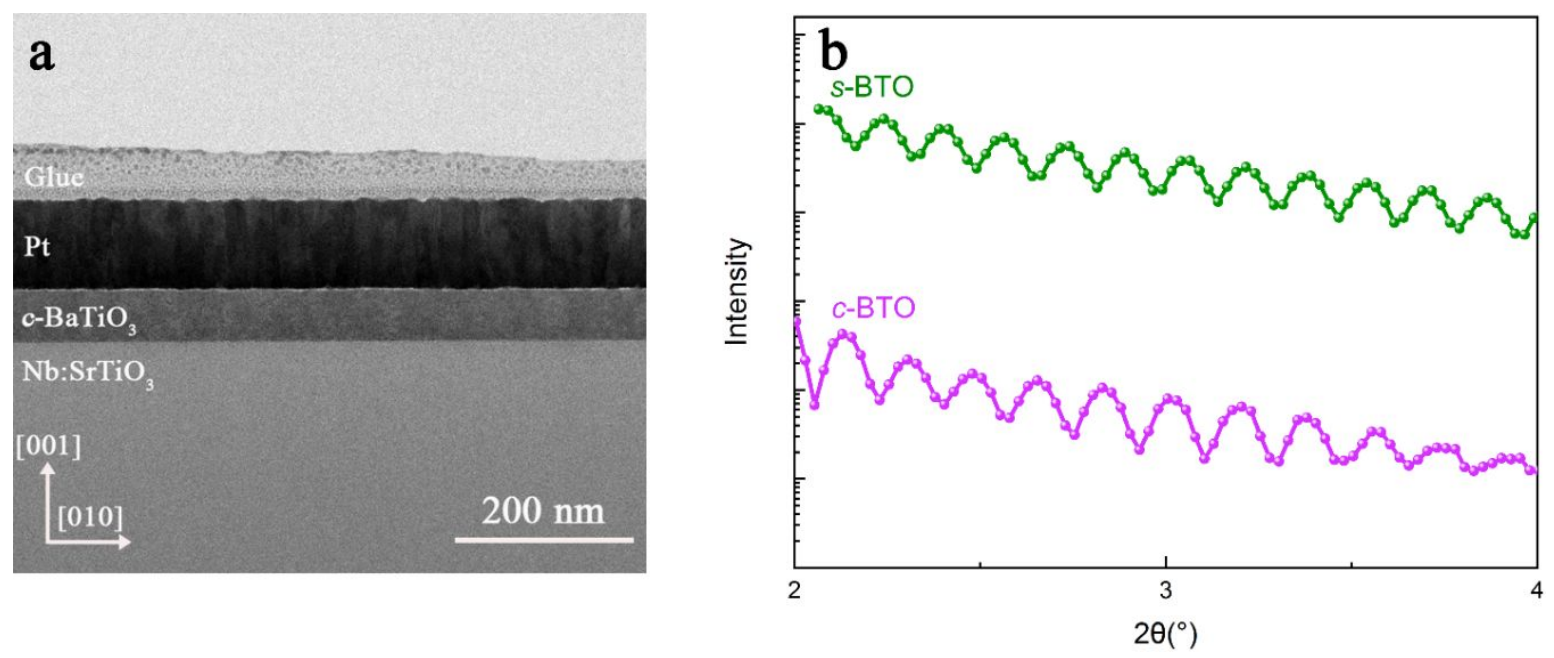

Figure S1. (a), the cross-sectional TEM image of $c$-BTO film and (b), The XRR of c-BTO and s-BTO with $2 \theta$ from $2^{\circ}$ to $4^{\circ}$.

The thickness of the single-layer film could be determined by the angular distance of two successive maxima in the XRR. The periods of oscillation of the two films are nearly the same, indicating the similar thickness. The calculated thicknesses of $c$-BTO and $s$-BTO films are 58.6 and $61.2 \mathrm{~nm}$, respectively, which coincide with the thickness in Figure S1a. ${ }^{1-3}$

\section{Defect and valence of the films}
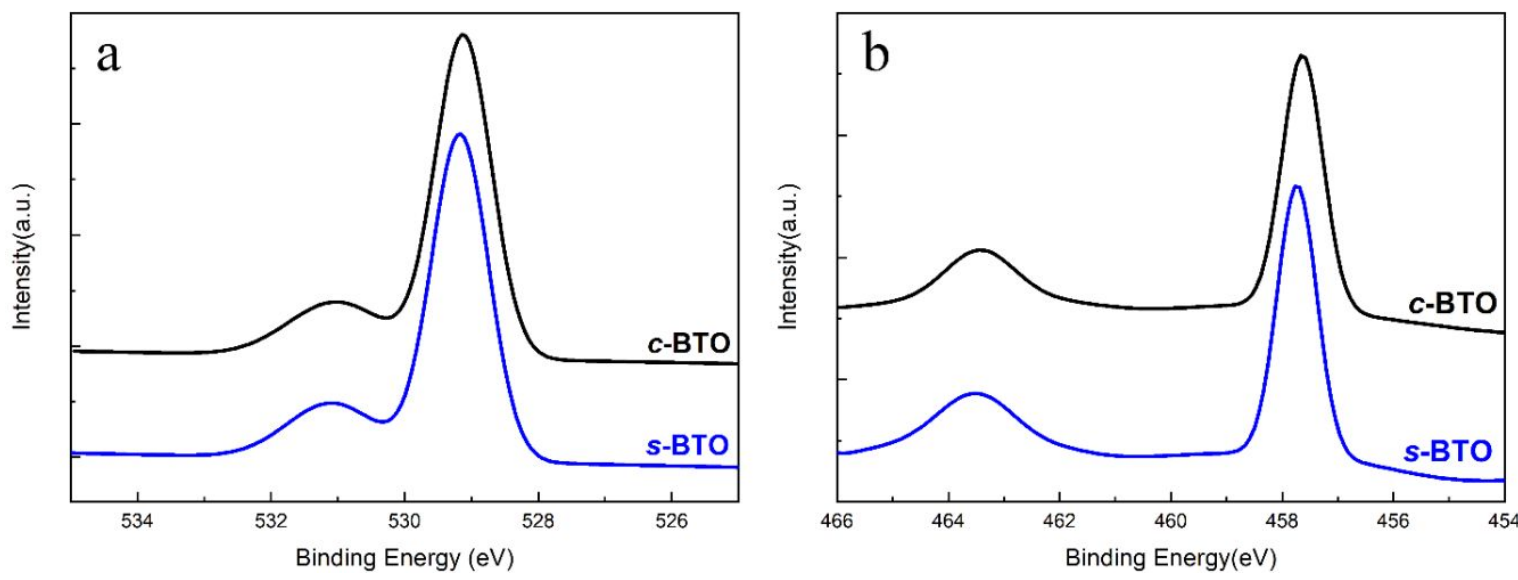

Figure S2. (a) O $1 s$ and (b) Ti $2 p$ XPS spectra of of $c$-BTO and $s$-BTO film. The main peak at $529.2 \mathrm{eV}$ corresponds to the lattice oxygen while the satellite peak at $531.1 \mathrm{eV}$ is regarded as the oxygen vacancies. The amount of the oxygen vacancies could be determined by the 
peak areas of the satellite peaks. The similar intensity and areas of the two films reveal that the amounts of oxygen vacancies are nearly the same. The peak positions in Ti $2 p$ XPS resembles and shows no indication of $\mathrm{Ti}^{3+}$ with lower binding energy, inferring that the domination of $\mathrm{Ti}^{4+}{ }^{4-6}$. Similar amount of oxygen vacancies as well as $\mathrm{Ti}^{4+}$ rule out the contribution of defect-dipoles to the enhancement of tetragonality ${ }^{7}$.

\section{Large-area AFM image}

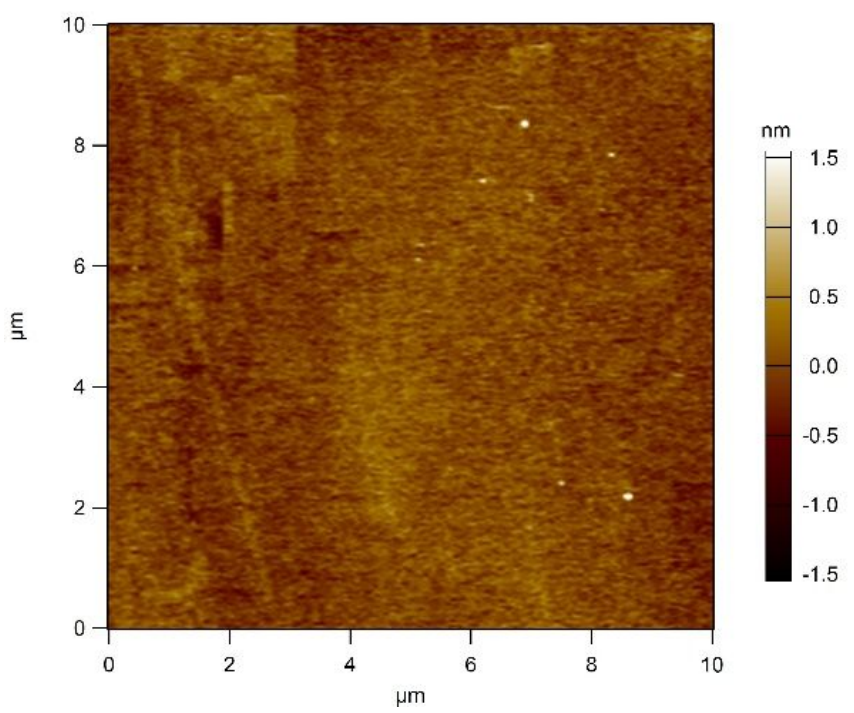

Figure S3. The AFM image of $c$-BTO with $10 \times 10 \mu \mathrm{m}^{2}$ areas. The smooth surface with minimal clusters and no grain boundary indicate the excellent quality and epitaxy of $c$-BTO. The minimal clusters are the common fault of ferroelectric oxides during deposition.

\section{Temperature-dependent lattice structures of $c$-BTO}
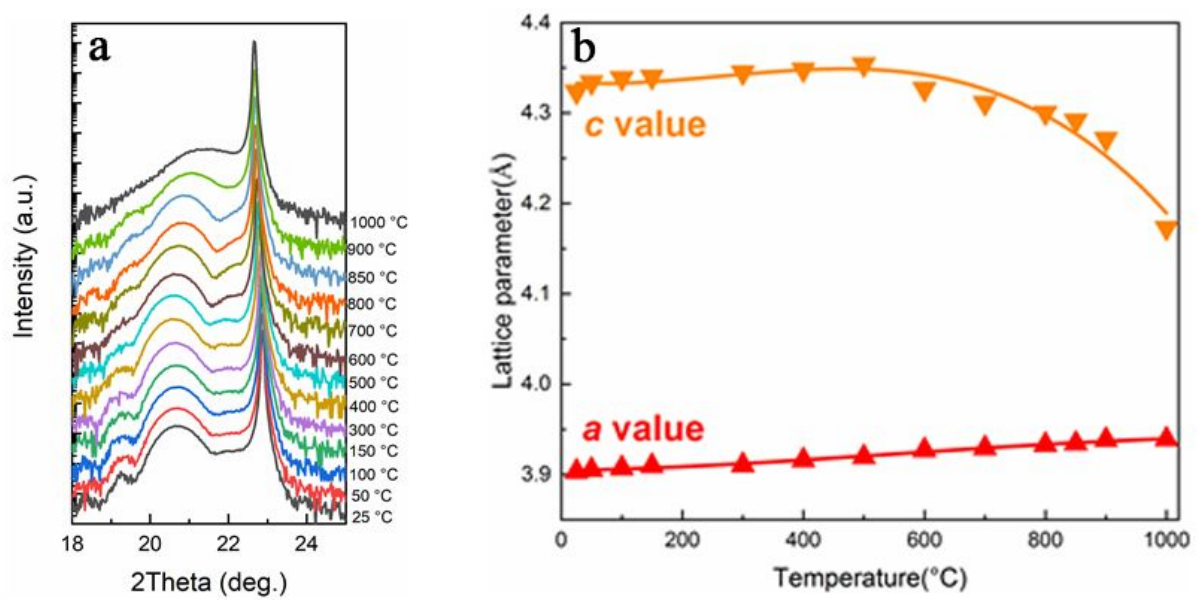
Figure S4. (a), the XRD evolution of the $c$-BTO around the (001) peaks with temperature. The peak position of $c$-BTO (001) tends to decrease until $500{ }^{\circ} \mathrm{C}$ then increases with rising temperature, without a kink reperesnt the phase transition point as literatures, ${ }^{8910}$ which indicates the highly stable ferroelectric phase. The peak of Nb:STO (100) shows monotone decreasing tendency The calculated lattice parameters of $c$-BTO dependent to the temperature are shown in (b), which proves the tetragonality keeps up to $1000{ }^{\circ} \mathrm{C}$.

\section{The RSMs of $c$-BTO at $600{ }^{\circ} \mathrm{C}$ and $1000{ }^{\circ} \mathrm{C}$}
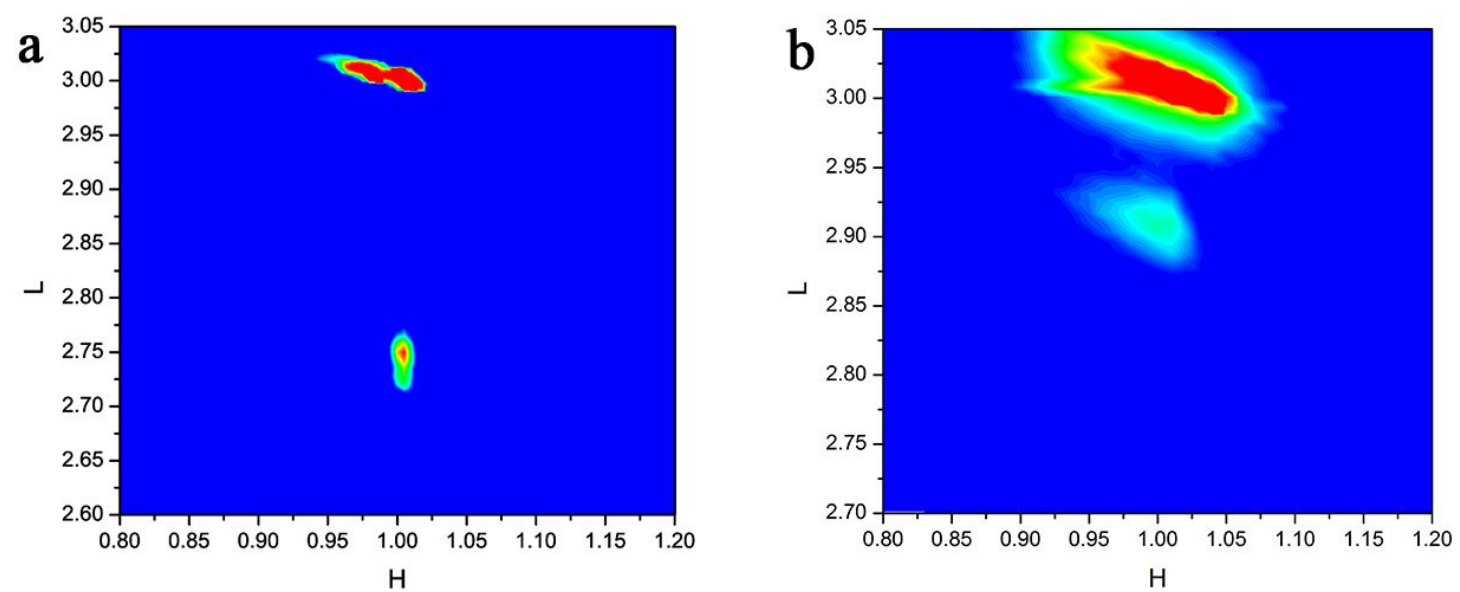

Figure S5. The RSM along (103) plane of $c$-BTO at (a) $600{ }^{\circ} \mathrm{C}$ and (b) $1000{ }^{\circ} \mathrm{C}$. The tetragonality could be maintained up to $1000{ }^{\circ} \mathrm{C}$, and the $c / a$ value is suppressed, which is consistent with the XRD results shown in Figure.S4a. Notably, the data are poorer than the room-temperature RSM in Figure.1, which is due to the high scanning rate for the consideration of the safety of the facility as well as avoiding the reaction of $\mathrm{BaTiO}_{3}$ during long time high-temperature scanning. But the RSMs are still clear enough to claim the tetragonality of $c$-BTO up to $1000^{\circ} \mathrm{C}$. 
Temperature-dependent hysteresis loops of $c$-BTO.

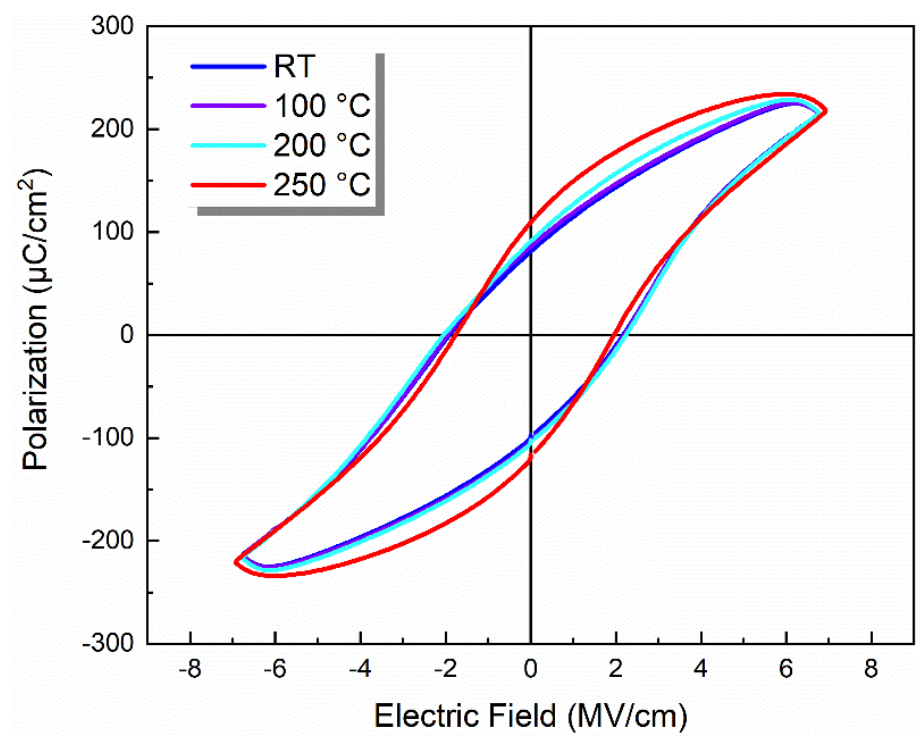

Figure S6. The temperature-dependent hysteresis loops of $c$-BTO film. Restricted by the limitation of the equipment, the temperature could only get to $250{ }^{\circ} \mathrm{C}$. Still, the profound ferroelectric nature of $c$-BTO at high temperature could prove the much increased Curie temperature.

The repeatable hysteresis loops of $c$-BTO.

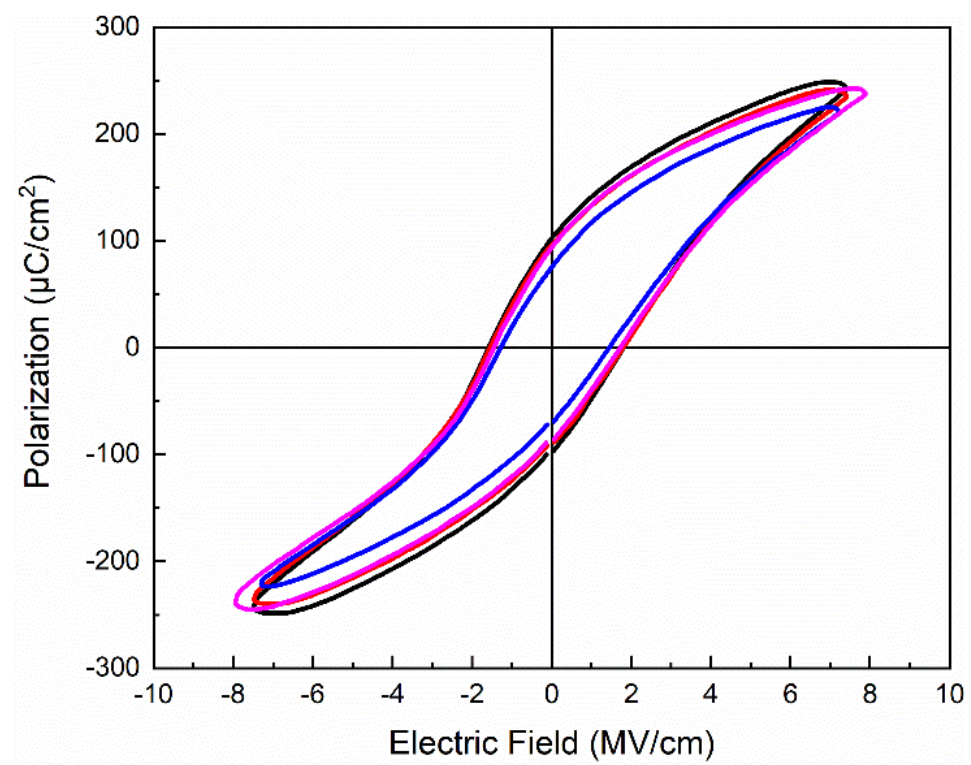

Figure S7. the repeatability of polarization measurements of $c$-BTO film. The hysteresis loops were measured by the same sample with different pads, showing good repeatability of the $c$-BTO film. 
The local phase hysteresis loop and the piezoresponse measurements

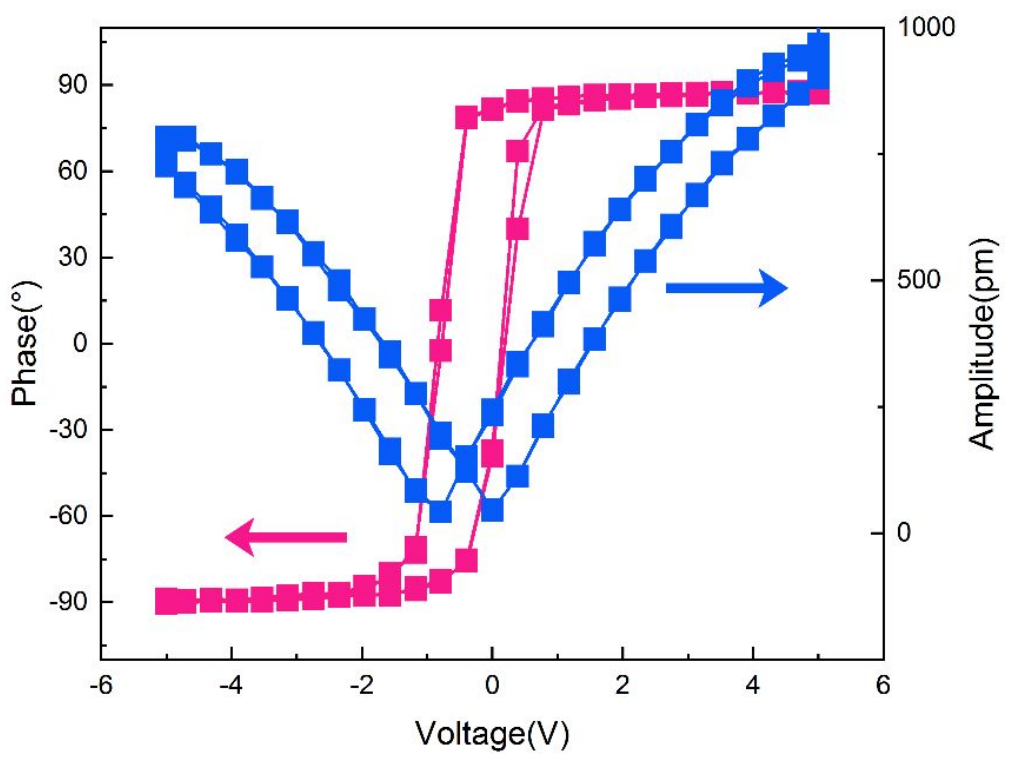

Figure S8. the local phase hysteresis loop and piezoresponse of $c$-BTO. The local phase and amplitude loops have been measured in various areas in Figure S3. All the loops show standard ferroelectric behaviors, even in the small cluster, meaning that the cluster is indeed simply the growth flaw rather than the $\mathrm{BaO}$ cluster.

The mapping of the polarization in HAADF-STEM.

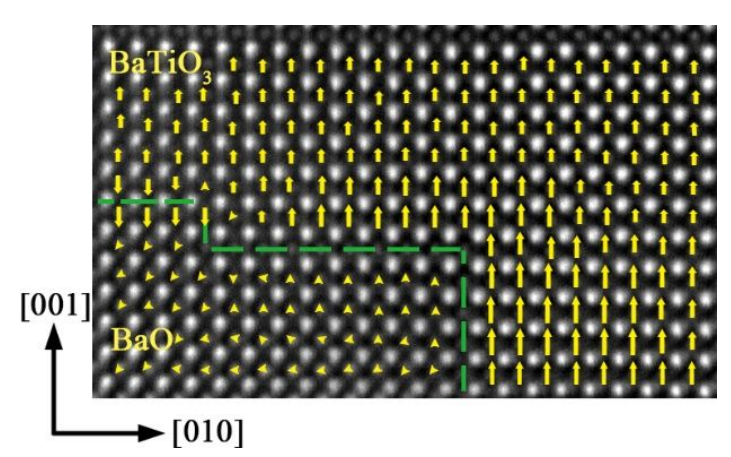

Figure S9. Superposition of the vectors of $\delta_{\mathrm{Ti}}$ in Figure 3c. The lengths of the yellow arrays represent $\delta_{\mathrm{Ti}}$. 


\section{The ICP results of $c$-BTO and $s$-BTO films.}

Table S1. The ICP result of $c$-BTO and $s$-BTO films.

\begin{tabular}{cccc}
\hline film & $\mathrm{Ba} /(\mu \mathrm{mol} / \mathrm{L})$ & $\mathrm{Ti} /(\mu \mathrm{mol} / \mathrm{L})$ & $\mathrm{Ba} / \mathrm{Ti}$ \\
\hline$c$-BTO & 0.194 & 0.108 & 1.80 \\
$s$-BTO & 0.182 & 0.166 & 1.09 \\
\hline
\end{tabular}

\section{References}

1 Ocko, B. M.; Wu, X. Z.; Sirota, E. B. X-ray reflectivity study of thermal capillary waves on liquid surfaces. Phys. Rev. Lett. 1994, 72, 242.

2 Parratt, L. G. Surface studies of solids by total reflection of X-rays. Phys. Rev. 1954, 95, 359.

3 Rhan, H.; Pietsch, U.; Rugel, S.; Metzger, H.; Persl, J. Investigations of semiconductor superlattices by depth-sensitive x-ray methods. J. Appl. Phys. 1993, 74, 146.

$4 \mathrm{Oku}, \mathrm{M}$; Wagatsuma, K.; Kohiki, S. Ti $2 p$ and Ti $3 p$ X-ray photoelectron spectra for $\mathrm{TiO}_{2}$, $\mathrm{SrTiO}_{3}$ and $\mathrm{BaTiO}_{3}$. Phys. Chem. Chem. Phys. 1999, 1, 5327.

5 Takatani, S.; Miki, H.; Kushida-Abdelghafar, K.; Torii, K. Pt/PbZr $\mathrm{Ti}_{1-x} \mathrm{O}_{3}$ interfacial reaction and Schottky barrier formation studied by x-ray photoelectron spectroscopy: Effect of $\mathrm{H}_{2}$ and $\mathrm{O}_{2}$ annealing. J. Appl. Phys. 1999, 85, 7784.

6 Zhu, T. J.; Lu, L. X-ray diffraction and photoelectron spectroscopic studies of (001)-oriented $\mathrm{Pb}\left(\mathrm{Zr}_{0.52} \mathrm{Ti}_{0.48}\right) \mathrm{O}_{3}$ thin films prepared by laser ablation. J. Appl. Phys. 2004, 95, 241.

7 Damodaran, A. R.; Enhancement of Ferroelectric Curie Temperature in $\mathrm{BaTiO}_{3}$ Films via Strain-Induced Defect Dipole Alignment. Adv. Mater. 2014, 26, 6341. 
8 Streiffer, S. K.; Eastman, J. A.; Fong, D. D. Observation of nanoscale $180^{\circ}$ stripe domains in ferroelectric $\mathrm{PbTiO}_{3}$ thin films. Phys. Rev. Lett. 2002, 89, 067601.

9 Choi, K. J.; Biegalski, M.; Li, Y. L. Enhancement of ferroelectricity in strained $\mathrm{BaTiO}_{3}$ thin films. Science 2004, 306, 1005.

10 Schlom, D. G.; Chen, L. Q.; Eom, C. B. Strain tuning of ferroelectric thin films. Annu. Rev. Mater. Res. 2007, 37, 589. 